Vol. 49 (1994) [349-350]

\title{
Determining shortest networks in the Euclidean Plane
}

\author{
J.F. WENG
}

The problem of constructing a shortest network which interconnects a given set of points with some extra points is usually called the Steiner problem, and correspondingly, the shortest network is referred to as a Steiner minimal tree [5]. Although this problem goes back three hundred years to Fermat, it really drew wide attention only after the sixties of this century [6]. Since it has been proved to be one of the NP-hard problems [4], we devote ourselves in this thesis to some fundamental techniques and their applications.

Chapter 1 is an introduction, giving terms, notations and some existing basic results.

Chapter 2 deals with the fundamental techniques. The indirect comparison based on the variational approach [8] is used to study Steiner minimal trees for any four points and to prove an embedding theorem. A method to construct general Steiner polygons [1] is presented. The concept of symmetrisation is introduced, and as a result, Pollak's theorem [7] becomes a simple corollary of our symmetrisation theorem.

In Chapter 3, the Steiner minimal trees for two extreme classes of sets are determined. One class is the set of zigzag lines for which we generalise an existing result [2]. The other class is the set of regular polygons with centre. This problem is much more complicated than the original one of the Steiner problem of regular polygons without centre. The latter problem is regarded as the first genuine instance of the Steiner problem in the history of the topic [3].

Finally, in Chapter 4, the decomposition problem is investigated; that is 'When is the Steiner minimal tree on a union of sets just a union of the Steiner minimal trees on these sets separately?' In particular, we reveal the structures of the Steiner minimal trees for consecutive orthogonal rectangles. These can be used to test the performance of heuristics of the Steiner problem.

Received 2nd November, 1993

Thesis submitted to the Flinders University of South Australia, June 1993. Degree approved: August 1993. Supervisor: Dr. R.S. Booth.

The scholarships awarded by the Australian Government and the Flinders University are gratefully acknowledged. The author also wishes to thank his supervisor Dr. Ray Booth for his constant advice and constructive criticism during the completion of the work.

Copyright Clearance Centre, Inc. Serial-fee code: 0004-9729/94 \$A2.00+0.00. 


\section{REFERENCES}

[1] E.J. Cockayne, 'On the efficiency of the algorithm for Steiner minimal trees', SIAM J. Appl. Math. 18 (1970), 150-159.

[2] D.Z. Du, F.K. Hwang and J.F. Weng, 'Steiner minimal trees on zigzag lines', Trans. Amer. Math. Soc. 278 (1983), 149-156.

[3] D.Z. Du, F.K. Hwang and J.F. Weng, 'Steiner minimal trees on regular polygons', Discrete Comput. Geom. 2 (1987), 65-87.

[4] M.R. Garey, R.L. Graham and D.S. Johnson, 'The complexity of computing Steiner minimal trees', SIAM J. Appl. Math. 32 (1977), 835-859.

[5] E.N. Gilbert and H.O. Pollack, 'Steiner minimal trees', SIAM J. Appl. Math. 16 (1986), 1-29.

[6] H.W. Kuhn, 'Steiner's problem revised', in Studies in optimization, (G.B. Dantzig and B.C. Eavas, Editors), Studies in Math. 10 (Math. Assoc. Amer., 1975), pp. 53-70.

[7] H.O. Pollak, 'Some remarks on the Steiner problem', J. Combin. Theory Ser. A 24 (1978), 278-295.

[8] J.H. Rubinstein and D.A. Thomas, 'A variational approach to the Steiner network problem', Annals Oper. Res. 33 (1991), 481-499. 